\title{
Impartialité et indépendance du juge : une question de genre ?
}

\author{
Stéphanie Hennette Vauchez \\ Professeure de droit public \\ Université Paris Ouest Nanterre \\ UMR 7074 Centre de théorie et analyse du droit \\ Equipe CREDOF
}

L'indépendance et l'impartialité comptent assurément parmi les vertus les plus universellement reconnues comme gouvernant les systèmes de justice. Parfois complétés par des références à la " compétence notoire ${ }^{1}$ exigée des juges ou à leur respect de la dignité des fonctions juridictionnelles ${ }^{2}$, ces deux principes définissent les caractéristiques premières de la profession judiciaire. L'indépendance (vis-à-vis des pouvoirs politiques, mais aussi économiques, religieux, etc.) permet de garantir la fonction protectrice de la justice, tandis que l'impartialité garantit l'équité et la justesse des décisions judiciaires - répressives ou non. C'est au nom de ces vertus cardinales que tout système de justice instaure des règles d'appartenance à la profession : règles de compétence pour y accéder, mécanismes disciplinaires permettant de sanctionner des manquements à la dignité des fonctions voire, de prononcer l'exclusion - de ceux qui y portent atteinte.

On souhaite ici interroger ces vertus judiciaires - très usuellement traduites, en termes techniques, dans les textes juridiques de nature diverse qui créent des juridictions ou définissent le statut des juges - qu'il s'agisse de constitutions, ordonnances, traités ou règlements intérieurs. Et on souhaite le faire, en écho à la problématique qui sous-tend le présent ouvrage, en prenant de la distance avec une compréhension de l'indépendance qui y verrait un ensemble de qualités morales exigibles des juges pour l'appréhender, bien plutôt, comme l'une des technologies politiques essentielles à la (re)légitimation de la figure du juge dans le monde contemporain où il semble appelé à jouer un rôle croissant ${ }^{3}$. La question que I'on pose ici est la suivante : y-a-t-il un genre de l'indépendance, de l'impartialité, de la " compétence notoire » (...) en tant que principes constitutifs des professions judiciaires? Un bref regard porté sur la composition sexuée de la justice à travers le monde amène en effet à poser la question : dès lors qu'on ne peut que constater la nette et très répandue sous-représentation des femmes dans les mondes judiciaires ${ }^{4}$, la question de savoir si ces

\footnotetext{
${ }^{1} \mathrm{CEDH}$, art. 21§1: "Les juges doivent jouir de la plus haute considération morale et réunir les conditions requises pour l'exercice de hautes fonctions judiciaires ou être des jurisconsultes possédant une compétence notoire $"$.

${ }^{2}$ V. par ex. décret $n^{\circ} 59-1292$ du 13 novembre 1292 du 13 novembre 1959 relatif aux obligations des membres du Conseil constitutionnel.

${ }^{3}$ Antoine Vauchez, " Penser la politique de l'indépendance », supra.

${ }^{4}$ C'est vrai à l'échelle des juridictions internationales sans aucun doute (Nienke Grossman, "Shattering the Glass Ceiling in International Adjudication ", Virginia Journal of International Law, 56, (2), 2016, p. 339) mais aussi très largement à l'échelle des juridictions suprêmes ou constitutionnelles nationales ainsi que, bien souvent, au cœur des ordres juridictionnels de droit commun nationaux. De manière intéressante, une telle sous-représentation des femmes tranche nettement avec le fait que, classiquement, les allégories de la justice la représentent sous des atours féminins : une femme les yeux bandés portant glaive et balance est le symbole classique comme contemporain de la justice.
} 
principes, en tant qu'ils constituent les exigences professionnelles et clefs d'accès à la profession judiciaire, ne jouent pas un rôle dans la perpétuation de la domination professionnelle masculine au sein de la justice, mérite d'être posée.

Historiquement, ils ont certainement joué ce rôle. Pensés, forgés et affirmés en des temps où les professions judiciaires - parmi de nombreuses autres - étaient en toute hypothèse interdites aux femmes, les principes d'indépendance et d'impartialité étaient assurément compris au prisme d'un fort biais de genre. II n'est qu'à penser, en termes analogiques, à la centralité de l'argument de la dépendance des femmes (notamment, en France, aux ministres du culte catholique) dans les discours s'opposant, jusqu'en 1944, à l'acquisition du droit de vote des femmes. L'enquête historique sur les professions de prestige menée par Juliette Rennes ${ }^{5}$ confirme en toute hypothèse cette intuition. Or les femmes ont aujourd'hui largement accès à toutes les professions - notamment, les professions juridiques ${ }^{6}$. II est même des pays où c'est désormais leur trop grande féminisation qui inquiète ${ }^{7}$. Est-ce à dire que le temps où indépendance et impartialité de la justice renvoyaient à des vertus prêtées aux hommes est révolu ? Une telle conclusion se heurterait à diverses objections. Une objection empirique, en premier lieu : bien que les chiffres de leur participation aux fonctions juridictionnelles de par le monde se soient considérablement accrus au cours des dernières décennies, elles demeurent largement sous-représentées - de sorte qu'il n'est pas exclu de penser que d'éventuels biais de genre perdurent dans la mise en œuvre des principes constitutifs de la justice que sont l'indépendance, l'impartialité et leurs corollaires. On peut d'ailleurs signaler que, depuis une dizaine d'années environ, cette manière précise d'interroger le genre de la justice a suscité l'émergence de travaux innovants, dans les champs de la sociologie et de la théorie politique notamment ${ }^{8}$. Une objection dynamique, en second lieu. L'observation et l'analyse des débats relatifs à la place des femmes dans la justice (position des acteurs, teneur de arguments échangés, etc.) permet en effet de mettre en évidence deux éléments qui structureront la présente contribution : l'un, épistémologique et l'autre, argumentatif.

\footnotetext{
${ }^{5}$ Juliette Rennes, Le mérite et la nature. Une controverse républicaine : l'accès des femmes aux professions de prestige 1880-1940, Paris, Fayard, 2007.

${ }^{6}$ Ce qui ne saurait signifier que l'égalité professionnelle entre hommes et femmes est acquise - loin de là. Simplement, dans un grand nombre de pays, les barrières formelles qui interdisaient autrefois l'accès de nombre de professions aux femmes ont, pour l'essentiel, disparu.

${ }^{7}$ La France est un exemple typique, où les femmes représentent actuellement (et depuis plusieurs années déjà) plus de 75\% des auditrices de l'Ecole nationale de la Magistrature (ENM). On trouve dans le rapport annuel du Conseil supérieur de la Magistrature pour 2014 les chiffres suivants concernant le stock de magistrats : 5192 femmes pour 3000 hommes en poste (soit $63,4 \%$ femmes et $36,6 \%$ hommes). Le plafond de verre demeure néanmoins défavorable aux femmes qui n'accèdent pas dans des proportions équivalentes aux postes hors hiérarchie. Sur la France, v. Mustapha Mekki (dir.), La féminisation de la justice, Paris, Economica, 2011. D'autres pays connaissent une féminisation comparable. On peut ainsi citer le cas du Portugal, où, bien que les femmes n'aient accès à la magistrature que depuis 1974, la féminisation est forte. En 2015, 59\% des 1990 magistrat-es des cours de $1^{\text {re }}$ instance, cours d'appel et cour suprême étaient des femmes. L'année précédente, $67,5 \%$ des auditeur-es de justice étaient des femmes. V. Madalena Duarte, Ana Oliveira, Paula Fernando, "Gender and Judging in Portugal: Opinions and Perceptions », Oñati Socio-legal Series [online], 6 (3), 2016, p. 477-495: http://ssrn.com/abstract=2831939.

${ }^{8} \mathrm{~V}$. par ex. les travaux de Sally Kenney, et notamment : Gender and Justice, Why Women in the Judiciary Really Matter, London, Routledge, 2012 ; ou encore, parmi diverses références: Ulricke Schultz, Gisela Shaw (dir.), Women in the Judiciary, London, Routledge, 2012 ; Nienke Grossman, " Sex on the Bench: Do Women Judges Matter to the Legitimacy of International Courts ? ", Chicago Journal of International Law, vol. 12, 2012, p. 647 et, plus récemment : Josephine Dawuni, Akua Kuenyehia, International Courts and the African Woman Judge: Unveiled Narratives, London, Routledge, 2017.
} 
D'un point de vue épistémologique, il est frappant de constater que le plus souvent, lorsque la question de la place des femmes est abordée au sein d'une arène judiciaire, elle suscite une très vive anxiété et déclenche des réactions et contre-mobilisations très fortes signe de tensions sous-jacentes. On s'appuiera sur quelques exemples récents, en France d'une part et à l'échelle du Conseil de l'Europe d'autre part, pour établir non seulement que la question de la composition sexuée des corps judiciaires n'est posée que difficilement, mais aussi que, quand elle l'est, elle génère de fortes anxiétés et contre-mobilisations (I). D'un point de vue de stratégie argumentative, on suggérera que ces crispations sont d'autant plus étonnantes, ou paradoxales, que la restructuration de nombre de systèmes et de théories démocratiques autour de la figure centrale du juge d'une part, et la souplesse heuristique de ces notions d'indépendance et d'impartialité d'autre part, pourraient au contraire fort bien se prêter à des interprétations qui rendraient possibles, voire requerraient, une représentation équilibrée des femmes et des hommes sur les bancs de la justice (II).

\section{Une question subversive}

L'expérience prouve que la question de la place des femmes dans la justice ou, plus généralement, de la représentation équilibrée entre les sexes des cours et juridictions de par le monde, apparaît éminemment subversive. Elle n'émerge que difficilement sous le poids des forces multiples qui cherchent à la disqualifier et l'empêcher; et lorsqu'elle parvient à s'inscrire à l'agenda, elle suscite typiquement des contre-mobilisations massives.

\section{A. L'égalité entre les sexes dans la justice : une question qui peine à émerger}

Les tensions autour de la question du sexe des juges se donnent à voir dans une diversité de juridictions, tant au niveau national qu'international. On s'attachera ici à deux exemples : celui de la magistrature française, via l'analyse de la mise de cette question à l'agenda du Conseil supérieur de la Magistrature (CSM), et celui de la justice internationale, via l'étude de la position sur ce point de l'Institut du droit international.

\section{Les femmes dans la magistrature française : retour sur une mobilisation récente du CSM}

S'il était déjà possible depuis plusieurs années de trouver, dans les rapports annuels du CSM, des statistiques sexuées sur la composition du corps des magistrats judiciaires, ce n'est qu'en 2011 (i.e. bien tardivement ${ }^{9}$ ) que des enseignements en sont tirés, et qu'est mise en évidence l'épaisseur du plafond de verre qui structure le corps des magistrats de l'ordre judiciaire en France. On découvre ainsi dans ce rapport de 2011 que, alors que depuis plusieurs années déjà, $80 \%$ des auditeurs de l'Ecole nationale de la Magistrature sont des femmes ${ }^{10}$, elles n'occupent qu'une faible part des postes les plus importants dans la

\footnotetext{
${ }^{9}$ Par comparaison : le New Jersey crée une task force sur les femmes dans la justice en 1983 ; au Canada et en Australie, des politiques volontaristes de diversité dans la justice sont mises en œuvre à partir des années 1990...

${ }^{10}$ Clairement, le système méritocratique du concours favorise les femmes, par opposition, par exemple, aux systèmes de cooptation. V. Anne Boigeol, "Les magistrates en France : des stratégies particulières ? ", in Anne
} 
magistrature. Sur la tranche d'âge des plus de 65 ans, seules $35 \%$ des femmes sont hors hiérarchie (contre 55\% des hommes); et sur la tranche d'âge de 55 à 59 ans, ce chiffre tombe à $14 \%$ des femmes (contre $26 \%$ pour les hommes). De même, l'étude des nominations à de hautes fonctions entre 2007 et 2011 révèle que seules 5 femmes ont été nommées sur 54 postes de premiers présidents de Cour d'appel, et seulement 50 sur les 195 postes de présidents de tribunaux de grande instance ${ }^{11}$. De la même manière, on ne compte que 38 femmes parmi les 197 procureurs de la République nommé-es de 2006 à $2011^{12}$.

De manière étonnante, la publication de ces chiffres et la mise en évidence du contraste entre la féminisation très forte et ancrée du corps en général et la persistance d'un épais plafond de verre a donné lieu à des mises en discussion paradoxales. En effet, largement à rebours du constat établi par les chiffres, les réponses et réactions dominantes au rapport de 2011 furent essentiellement articulées autour de la double idée que, d'une part, l'évolution " naturelle » était favorable à un " rattrapage " et que, d'autre part, tout dispositif volontariste était par définition impensable dans la magistrature, en raison du principe de l'indépendance de la justice. Par le biais de son rapport pour l'année 2012, le CSM exprime ainsi son hostilité à l'hypothèse d'une application à la magistrature de la loi Sauvadet du 12 mars 2012, qui crée des quotas par sexe (de 40\% à horizon 2018) pour les nominations dans les " emplois supérieurs " de la fonction publique ${ }^{13}$. Le CSM souligne ainsi en premier lieu le caractère inutile ou superfétatoire de l'application d'un dispositif contraignant tel que celui imaginé par la loi Sauvadet. Insistant sur l'importance qui s'attache au fait de raisonner "en flux" et non " en stock", le Conseil met en avant l'argument de la résolution " naturelle » du déséquilibre entre les sexes : soulignant qu'elles représentent (déjà ?) " en 2012 39,9\% de l'ensemble des emplois hors hiérarchie " au siège (mais seulement $22,5 \%$ au parquet...), il semble suggérer que les objectifs de la loi Sauvadet sont en fait déjà atteints (même si c'est "à un degré moindre " au parquet ${ }^{14}$ ). Plus vigoureusement encore, le CSM exprime ensuite son opposition de principe à un tel mécanisme. C'est en effet le principe d'indépendance de l'institution judiciaire qui impose de mettre en cause " la légitimité d'une telle intervention du pouvoir législatif ${ }^{15}$. En cela, la magistrature se différencie des autres sphères de la haute fonction publique que la loi Sauvadet a voulu repenser au prisme de l'égalité entre les sexes :

"L'extension de la logique de la loi Sauvadet à la magistrature serait pourtant problématique en ce que cette loi a été conçue pour viser, de façon pérenne, des choix discrétionnaires au sein d'un vivier quasiment extensible à volonté. S'agissant des emplois dits "à la discrétion du gouvernement", les conditions d'âge et d'expérience pour être nommé préfet ou ambassadeur

Devillé, Olivier Payre (dir.), Les femmes et le droit. Constructions idéologiques et pratiques sociales, Bruxelles, Publications des facultés universitaires Saint Louis, 1999, p. 151.

${ }^{11}$ Ces chiffres, extraits du Rapport annuel 2011, sont complétés par une autre présentation tout aussi frappante que l'on trouve dans le Rapport annuel 2012, p. 195 : « 75 \% des magistrats du second grade sont des femmes et la circonstance que près de $75 \%$ des présidents de TGI et des premiers présidents de cour d'appel sont des hommes (et la présence des hommes est encore plus forte dans les plus hauts postes au parquet) ".

12 C. Girard, "Entretien avec Martine Lombard", Revue des droits de l'Homme, 2012, $\mathrm{n}^{\circ} 2$ : https://revdh.revues.org/182

${ }^{13}$ O. Bui Xuan, "L'égalité professionnelle entre hommes et femmes dans la fonction publique : une révolution manquée ? ", Actualité juridique Droit administratif, 2012, p. 1100.

${ }^{14}$ CSM, Rapport annuel 2012, p. 223.

${ }^{15}$ Ibid., p. 196. 
sont en effet, en droit, très souples. Ce n'est pas le cas de la magistrature qui, par son indépendance, n'est assimilable ni à la préfectorale, ni à la diplomatie notamment. Le vivier des fonctions judiciaires aux plus hauts niveaux n'est pas "à la discrétion du gouvernement" (...) ; il est encadré strictement par le statut : seuls des magistrats peuvent être nommés à ces postes, à condition qu'ils aient émis des desiderata en ce sens ${ }^{16}$.

En outre, et parallèlement à ces réserves, l'émergence de la question de l'égalité entre les sexes au sein de la magistrature française a fait émerger un certain nombre d'anxiétés relatives à la situation de déséquilibre entre les sexes qui caractérise désormais et pour un certain temps au moins- la magistrature française. En 1999 (époque à laquelle les femmes représentent déjà la moitié du corps), Anne Boigeol observait: "Sans être présentée comme un enjeu majeur pour la profession, la forte féminisation suscite un malaise, des craintes, des interrogations sur les raisons du phénomène, ses conséquences, les problèmes qu'il est susceptible d'engendrer $"{ }^{17}$. Il est, dans ces conditions, intéressant de souligner que, dans le courant des années 2000, la mission de recherche Droit \& Justice (sur la politique scientifique de laquelle l'ENM comme les différentes directions du ministère de la Justice ont une voix qui pèse) finançait une recherche consacrée à " La féminisation de la justice ${ }^{18}$. Participe possiblement encore de cette inquiétude face à une féminisation trop forte du corps le fait que l'ENM confiait en 2011 à un laboratoire de psychologie sociale une enquête sur la moindre attractivité de la magistrature ${ }^{19}$ - à l'occasion de laquelle féminisation et moindre attractivité étaient ici significativement mises en équivalence. Plus largement, et au-delà même du monde judiciaire, on observe une mise en cause inédite et croissante de l'impact du nombre sans cesse (fatalement) croissant des formations de jugement composées uniquement de femmes sur la qualité et la légitimité de la justice. On songe par exemple aux mobilisations initiées par des groupes de pères (à l'instar de l'association SOS papas) mettant en cause des juridictions aux affaires familiales ${ }^{20}$. On se souvient ainsi, au cours de l'année 2013 , des happenings organisés par des pères grimpant au sommet de grues pour attirer l'attention sur le faible nombre d'entre eux qui se voient confier la garde de leurs enfants en cas de séparation. Ces mobilisations et happenings ont reçu un fort écho médiatique.

En d'autres termes, ce à quoi on assiste suite de la mise à l'agenda de la question du déséquilibre entre les sexes au sein de la magistrature, c'est d'une certaine manière l'inversion de la question: c'est la question de l'impact de formations de jugement composées uniquement de femmes sur la qualité ou la légitimité de la justice qui émerge, alors même que la situation précédente (i.e. la longue et large prédominance de formations de jugement composées exclusivement d'hommes) n'avait jamais posé de problème. II s'agit là d'un phénomène classique de backlash ; ainsi, au Canada ou aux Etats-Unis, la littérature recense de nombreux cas où l'accès d'un-e juge d'une certain-e minorité (sexe, race, religion, etc.) à des fonctions judiciaires se traduit par une mise en cause de l'impartialité

\footnotetext{
${ }^{16}$ Ibid., p. 224 (nous soulignons).

${ }^{17}$ Anne Boigeol, "Les magistrates en France : des stratégies particulières ? ", art. cité

${ }^{18}$ Mustapha Mekki (dir.), La féminisation de la justice, op. cit.

${ }^{19}$ Rapport de Rémi Finkelstein, La féminisation de la magistrature : une exploration de quelques antécédents psychosociologiques, Université Paris-Ouest Nanterre La Défense, ENM, 2012.

${ }^{20}$ V. Marie Vogel, Anne Verjus, "Le droit des pères à faire famille: des mobilisations pour des droits nouveaux... sans obligation nouvelle ", in Agnès Martial (dir.), Des pères en solitaire ? Ruptures conjugales et paternité contemporaine, Aix-en-Provence, Presses de l'Université de Provence, 2016, p. 139-15 ; Aurélie FillodChabaud, "Les groupes militants de pères en France ", Informations sociales, n¹76, 2013.
} 
soudain menacée de la juridiction - surtout lorsque sont en cause des affaires de liberté religieuse, discrimination raciale ou de sexe... ${ }^{21}$. Certes, le cas national français est particulièrement emblématique, dès lors que c'est une situation très rare - à savoir : la très forte féminisation de la justice - qui fait naître le débat. Le problème se pose généralement dans l'autre sens. Tandis que dans de nombreux pays, c'est pour lutter contre les résistances à une facilitation de l'accès des femmes à la profession qu'il faut déployer du volontarisme politique, c'est, en France, le nécessaire renforcement de l'attractivité du métier pour les hommes qui fait figure d'objectif premier - suivi, pour certains acteurs, d'une réflexion sur les outils de lutte contre le plafond de verre qui cantonne les nombreuses femmes dans les postes moyens et inférieurs du corps. Mais cette spécificité est très intéressante, qui permet précisément de montrer que c'est bien le fait de poser la question du genre de la justice qui est subversif - indépendamment du sens dans lequel elle est posée ou de la situation de départ. Car en effet, ici comme ailleurs, on observe que la mise en cause, dans un sens ou dans un autre, d'un état du corps (et donc, du genre de l'indépendance) qui cause anxiétés et résistances.

\section{Les femmes et la justice internationale : les standards de l'Institut de droit international}

A l'échelle internationale, c'est depuis un peu plus d'une décennie que la question de la représentation équilibrée des hommes et des femmes prend de l'importance. La nomination de Rosalyn Higgins, en 1995, comme première femme juge à la Cour internationale de justice a certainement constitué une étape-clef dans l'émergence de cette question; et, depuis le début des années 2000, on voit certains systèmes judiciaires internationaux formaliser leur réflexion, voire des règles, en la matière ${ }^{22}$. En 2011, l'Institut de droit international (IDI) - association scientifique indépendante créée en 1873, I'IDI est l'une des enceintes les plus traditionnelles du droit international - consacrait un rapport à "La situation du juge international ». Evoquant la question de la pertinence de la prise en compte du critère du sexe dans les règles de nomination des juges et de composition des juridictions, le rapport est dépourvu de toute équivoque - et de toute souplesse. II rappelle en premier lieu l'esprit des dispositions portant statut de la Cour internationale de justice :

« La Cour est un corps de magistrats, indépendants élus, sans regard à leur nationalité, parmi les personnes jouissant de la plus haute considération morale et qui réunissent les conditions requises pour l'exercice, dans leurs pays respectifs, des plus hautes fonctions judiciaires, ou qui sont des jurisconsultes possédant une compétence notoire en matière de droit international "; ainsi que: "Dans toute élection, les électeurs auront en vue que les personnes appelées à faire partie de la Cour non seulement réunissent individuellement les conditions requises, mais assurent dans l'ensemble la représentation des grandes formes de civilisation et des principaux systèmes juridiques du monde ».

Mais lorsqu'il en vient, ensuite, à examiner les évolutions observables dans les statuts de certaines juridictions internationales visant, précisément, à promouvoir une représentation

\footnotetext{
${ }^{21}$ V. en ce sens : Maryka Omatsu, "The Fiction of Judicial Impartiality », Canadian Journal of Women and Law, 9 (1), 1997.

${ }^{22}$ Ainsi par exemple, la Cour pénale internationale instituée par le traité de Rome en 1998 est d'emblée définie comme une juridiction paritaire. Un objectif comparable est formulé à propos de la Cour africaine des droits de l'Homme et des peuples (créée en 1998); et le cas de la Cour européenne des droits de l'Homme est aussi intéressant, où un objectif de représentation équilibrée entre les sexes est formulé depuis 2004 (voir infra).
} 
accrue de magistrats de sexe féminin, le rapport se fait sévère. II fait ainsi référence au statut de Rome créant la CPI, qui précise que les Etats parties doivent tenir compte « de la nécessité d'assurer dans la composition de la Cour criminelle internationale une représentation équitable des hommes et des femmes " (art. 36§8(a)III), ou encore à une résolution de l'Assemblée parlementaire du COE de 2004 exigeant des Etats parties à la CEDH qu'ils fassent figurer, sur chaque liste de trois candidat-es qu'ils présentent pour remplir les fonctions de juge à la Cour européenne des droits de l'Homme, des candidat-es des deux sexes. Aussitôt, l'Institut de droit international exprime ses réserves par rapport à de tels mécanismes: "La stature morale et la compétence doivent rester les premiers critères de choix (...). Cette exigence doit l'emporter sur toute considération de sexe, $d^{\prime}$ 'origine ethnique ou de religion ${ }^{23}$. Aussitôt après, I'IDI met en avant l'argument de l'évolution "naturelle »: "Aussi bien les femmes, dans beaucoup de pays, sont-elles actuellement nombreuses à occuper des fonctions judiciaires de plus en plus importantes et ce mouvement au plan national se traduira tout naturellement au plan international dans les années à venir ${ }^{24}$. La résolution sur laquelle déboucha cette étude est à l'avenant, qui exprime l'idée que les procédures de sélection des juges internationaux doivent permettre d'assurer "la sélection de candidats de haut caractère moral, compétence et expérience, sans aucune discrimination notamment sur la base du sexe, de l'origine ou des croyances $" 25$. L'IDI s'oppose donc nettement aux mécanismes émergeants au sein des juridictions nationales, et qui reposent sur la prise en compte du sexe des juges nommés.

\section{B. L'égalité des sexes dans la justice : contre-mobilisations et backlash}

Les deux exemples précédents permettent donc de souligner combien la question de l'égalité entre les sexes peine à émerger dans le monde judiciaire - national comme international. II est frappant que lorsque, néanmoins, cette question est hissée en bonne place des agendas réformateurs de telle ou telle organisation judiciaire, elle suscite des contre-mobilisations puissantes - à la hauteur des tensions et anxiétés que fait naître le fait même de poser la question du genre de la justice. C'est ici sur l'exemple du Conseil de l'Europe que l'on souhaiterait fonder l'analyse.

En 2004, I'Assemblée parlementaire du Conseil de l'Europe adoptait une résolution indiquant que, pour ce qui est de l'élection des juges à la Cour Européenne des droits de l'Homme, elle ne prendrait plus en considération «les listes de candidats : [...] 3.2. ne comportant pas au moins un candidat de chaque sexe ${ }^{26}$. Or l'intensité et la pérennité des contre-mobilisations suscitées par cette nouvelle règle en font un exemple particulièrement emblématique, tant il existe de disproportion entre le caractère faiblement contraignant de la règle et les réactions qu'elle a suscitées.

Il y a autant de juges à la Cour européenne des droits de l'Homme (CEDH) que d'Etats membres du Conseil de l'Europe ; et lorsque vient son tour de procéder à la nomination d'un juge, chaque Etat doit soumettre à l'APCOE une liste de trois candidat-es, parmi lesquels I'Assemblée décide suite à un vote. Depuis 2004, chaque Etat doit donc veiller à ce que figure sur la liste de trois noms qu'il soumet au moins une femme (ou un homme). En outre,

\footnotetext{
${ }^{23}$ Souligné par moi.

${ }^{24}$ Souligné par moi.

${ }^{25}$ Institut de droit international, 6 RES EN FINAL, 9 sept. 2011, Session de Rhodes, The Position of the International Judge, $§ 5$.

${ }^{26}$ Résolution 1366, Candidats à la Cour européenne des droits de l'Homme, 30 janvier 2004.
} 
rien n'oblige l'Etat à présenter des candidats nationaux, de sorte que la contrainte qui pèse sur les Etats est en fait simplement l'exigence de trouver, sur la surface du globe, au moins une femme remplissant les qualités pour exercer les fonctions de juge à la CEDH. Ainsi rapportée au mode de désignation des juges à la $\mathrm{CEDH}$, la règle votée par l'Assemblée parlementaire du Conseil de l'Europe (APCOE) en 2004 ne peut être considérée, au mieux, que comme une forme faible de discrimination positive ${ }^{27}$. II n'en reste pas moins que cette forme faible de contrainte a suscité des formes extraordinaires, remarquables et effectives, de contre-mobilisation.

La contre-mobilisation a d'abord pris la forme classique de de la résistance - certains Etats refusant tout bonnement de se soumettre. L'Etat de Malte, d'emblée, refusera de se conformer à la nouvelle règle et présentera, par deux fois (en 2004 et en 2006), des listes composées uniquement d'hommes. Parallèlement, l'Etat de Malte n'aura de cesse de remettre en cause la légitimité de la nouvelle règle votée par l'APCOE et de revendiquer, à tout le moins, le nécessaire aménagement de "circonstances exceptionnelles " pouvant expliquer l'impossibilité dans laquelle se trouverait un Etat d'identifier une candidate idoine.

La contre-mobilisation se fait ensuite, de manière extraordinaire ou en tous cas jusqu'alors inédite, sous la forme de l'activation d'un mode d'intervention de la CEDH : I'avis consultatif. La Cour dispose en effet depuis 1970 d'une compétence consultative qui lui permet de donner son avis sur le sens à donner à telle ou telle disposition de la Convention européenne des droits de l'Homme. Elle n'en avait toutefois jamais usé, et il faut donc comprendre que la question de l'admissibilité du nouveau critère imposant aux Etats de lister impérativement des candidat-es des deux sexes a été perçue comme suffisamment importante pour qu'une procédure de facto extraordinaire fût actionnée. En l'occurrence, la Cour se rallie dans son avis consultatif à l'opinion maltaise selon laquelle il est nécessaire, à tout le moins, d'aménager une voie de sortie permettant aux Etats qui auraient fait tous les efforts requis mais n'auraient pas trouvé de femme valable à lister de soumettre des listes exclusivement composées d'hommes ${ }^{28}$.

Fortes et inédites, ces contre-mobilisations sont par ailleurs couronnées de succès, puisque la résolution de l'APCOE de 2004 qui avait mis le feu aux poudres sera altérée, en profondeur et en deux temps. La résolution est tout d'abord amendée, suite à un travail de mobilisation et de ralliement d'une majorité de membres de l'APCOE. Le texte de la nouvelle résolution votée en 2008 prévoit que : « L'Assemblée décide de prendre en considération les listes de candidats d'un seul sexe, si ces candidats appartiennent au sexe surreprésenté à la Cour, dans les cas exceptionnels où une Partie contractante a pris toutes les mesures nécessaires et adéquates pour garantir la présence du sexe sous-représenté sur la liste, mais n'a pas été en mesure de trouver un candidat de ce sexe qui satisfasse aux exigences du

\footnotetext{
${ }^{27}$ Margaret Thornton, " Otherness on the Bench : How Merit is Gendered ", Sydney Law Review, 29 (3), 2007, p. 406.

${ }^{28}$ Avis $n^{\circ} 1$ de la Cour, élection des juges, février $2008:$ « 54 : la Cour estime que [...] en ne permettant aucune exception à la représentation du sexe sous-représenté, la pratique actuelle de l'Assemblée parlementaire n'est pas conforme à la Convention : là où une Partie contractante a pris toutes les mesures nécessaires et adéquates en vue d'assurer la présence du sexe sous-représenté sur sa liste mais sans succès, et à plus forte raison quand elle a suivi les recommandations de l'Assemblée préconisant une procédure ouverte et transparente avec appel à candidatures (...), l'Assemblée ne saurait rejeter la liste en question pour la seule raison que cette présence n'est pas réalisée. II faut dès lors que des exceptions au principe de la présence obligatoire d'un candidat du sexe sous-représenté soient formulées dès que possible. »
} 
paragraphe 1 de l'article 21 de la Convention européenne des droits de l'homme ${ }^{29}$. La nouvelle formule est donc une version significativement altérée du principe de 2004, puisqu'elle aménage une large voie de sortie aux Etats récalcitrants, leur permettant d'arguer de circonstances exceptionnelles les menant à ne pouvoir lister que des hommes, tous leurs efforts pour trouver des candidatures féminines valables ayant échoué. Altérée, la règle est en outre vite durcie et formalisée par sa reprise dans les règles directrices du Comité des ministres, en $2012^{30}$.

Presque aussitôt, les conséquences de cette atténuation de l'exigence de listes comprenant au moins une femme se sont faites sentir : on voit reprendre la pratique des listes composées exclusivement d'hommes. C'est la Belgique qui ouvre le ban en 2011, mettant en avant le motif que, au moment précis où la liste était présentée, le seuil de $40 \%$ de femmes était tout juste atteint à la Cour de Strasbourg, de sorte qu'aucun sexe n'était plus sous-représenté et qu'en conséquence, la nouvelle règle instaurée par l'APCOE n'avait pas à s'appliquer. La Moldavie emboîte le pas en 2012, au motif qu'en dépit de tous les efforts de communication (appel public à candidatures, etc.), la seule femme candidate ayant présenté sa candidature était nettement moins qualifiée que ses homologues masculins. Depuis lors, le bilan des élections aux postes de juge à la Cour européenne des droits de l'Homme confirme le relativement faible effet de la règle : si en 2013, il y a deux hommes et deux femmes parmi les nouveaux juges de la Cour, la seule nouvelle recrue de 2014 est un homme, 7 des 11 recrues de 2015 sont des hommes, tandis qu'en 2016 comme en 2017, on compte 4 nouveaux juges, parmi lesquels une seule femme.

On voit donc ici, en prenant appui sur un exemple précis, combien un règle somme toute peu contraignante peut, lorsqu'elle touche comme ici à un sujet sensible, susciter des résistances et contre-mobilisations puissantes. En l'espace de quelques années, la règle de 2004 a donc été considérablement affaiblie : elle ne joue plus que lorsqu'un des deux sexes à la Cour est sous-représenté (moins de $40 \%$ ), et il est consenti aux Etats la possibilité d'y déroger lorsque des "circonstances exceptionnelles " ont rendu impossible l'identification d'une candidate féminine de qualité. En d'autres termes, si le nombre de femmes a progressé à la Cour de Strasbourg (pour atteindre, en novembre 2017, la paritéc ${ }^{31}$ ), le système semble internaliser la règle selon laquelle un déséquilibre en faveur des femmes serait impensable.

\section{Une question évitée}

Les considérations qui précèdent poussent donc bien à considérer qu'il y a quelque chose comme un genre de l'indépendance - qui se traduit tant par les chiffres (la fréquemment très nette domination des hommes sur les femmes dans le monde des cours

\footnotetext{
${ }^{29}$ Résolution 1627, Candidats à la Cour européenne des droits de l'Homme, 30 sept. 2008 ; souligné par moi. $30 \quad \mathrm{CM}(2012) 40 \quad$ final

https://wcd.coe.int/ViewDoc.jsp?Ref=CM\%282012\%2940\&Language=lanFrench\&Ver=final\&Site=CM\&BackCol orInternet=C3C3C3\&BackColorIntranet=EDB021\&BackColorLogged=F5D383 : «8. Les listes des candidats devraient, en règle générale, comprendre au moins un candidat de chaque sexe, sauf si les candidats appartiennent au sexe sous-représenté à la Cour (moins de $40 \%$ des juges) ou lorsque des circonstances exceptionnelles conduisent à une dérogation à cette règle $"$.

${ }^{31} 23$ femmes sur 47 postes de juge.
} 
et tribunaux de par le monde) que par les efforts déployés pour maintenir cet état des choses et résister à toute évolution vers des exigences de représentation équilibrée des femmes et des hommes dans le monde de la justice. En ce sens, le genre fait bien figure de bouclier du bastion masculin qu'est la justice. Ceci tranche singulièrement avec d'une part, le rôle désormais joué par la justice dans les démocraties contemporaines et d'autre part, la montée en puissance d'outils de vérification de l'indépendance et de l'impartialité telles que la théorie des apparences. Au plan théorique en effet, le principe même du constitutionnalisme comme l'aménagement concret des garanties offertes, en démocratie libérale, par le standard du procès équitable semblent en effet pouvoir être aisément conciliés avec les exigences de représentation équilibrée entre les sexes au sein du monde de la justice - ce qui ne rend que plus nette encore la dimension de résistance active qui caractérise le sort réservé par nombre d'ordres juridiques à cette revendication émergente : la question est évitée. En ce sens, si « la justice » compte assurément parmi ceux des objets étudiés dans le présent ouvrage pour lequel l'indépendance est à la fois une vertu et une exigence anciennes, il apparaît que ce monde judiciaire n'est pourtant pas indemne de la redécouverte de l'indépendance qui, depuis les années 1990, la voit se déployer partout où émergent les figures nouvelles du régulateur -du banquier central à l'arbitre en passant par le déontologue. L'indépendance de la justice est, en effet, largement travaillée par certaines revisitations de l'idée de représentativité -laquelle est, en amont, altérée par la montée en puissance du paradigme de la représentation équilibrée en les sexes.

\section{A. Centralité de la figure du juge dans le paradigme constitutionnaliste : responsabilité et représentativité}

La conception même du juge et du rôle qu'il joue dans les démocraties contemporaines constitue probablement l'un des terrains sur lesquels la théorie du droit a connu les évolutions les plus profondes au cours du dernier siècle ou demi-siècle ${ }^{32}$. La figure du juge "bouche de la loi » est bien définitivement considérée comme dépassée, et différentes écoles théoriques de pensée (normativistes, réalistes, etc.) acceptent aujourd'hui très largement le rôle créateur et, pour tout dire, politique, du juge. Les explications et justifications sont, bien sûr, différentes. Ainsi, alors que la tradition kelsenienne va qualifier le contrôle juridictionnel de la Constitution comme un acte de co-législation (et résoudre ainsi la question de l'antinomie entre volonté générale et contrôle de la loi), la tradition réaliste concentre son analyse sur le pouvoir $d$ 'interprétation des règles juridiques nécessairement créateur au plan normatif -, ce qui fait du juge non plus une autorité d'application, mais de création, du droit. Ces distinctions méritent, bien sûr, d'être affinées et complétées ; mais on les évoque ici simplement pour souligner leur convergence autour de l'idée que le juge est devenu une figure centrale des démocraties constitutionnelles. On soulignera encore que, prospérant sur ces bases, le courant théorique contemporain du constitutionnalisme global (ou néoconstitutionnalisme ${ }^{33}$ ) fait de l'idée que « la démocratie libérale serait incomplète et déficiente sans judicial review [contrôle juridictionnel de la

\footnotetext{
32 Jacques Krynen, L'Etat de justice, France, XIIlè-XXè siècle. L'emprise contemporaine des juges, tome 2, Paris, Gallimard, 2012.

${ }^{33}$ Sur ces deux notions - et ce qui les lie -, on renvoie à Mauro Barberis, « Le néoconstitutionnalisme existe-til ? ", Revus - Journal for constitutional theory and philosophy of law, n²5, 2015, p. 101. V. aussi Alec Stone Sweet, Jud Matthews, "Proportionality Balancing and Global Constitutionalism ", Columbia Journal of Transnational Law, vol. 47, 2008.
} 
législation] ${ }^{34}$ un leitmotiv. Même, le judicial review fait parfois figure de composante essentielle de la notion même de démocratie représentative, laquelle se voit redéfinie par la théorie constitutionnelle contemporaine comme reposant sur (ou supposant) une délégation de pouvoir supplémentaire par rapport aux "vieilles " théories classiques de la démocratie représentative. Un auteur comme Mathias Kumm explique ainsi que, tout comme le passage de la démocratie directe athénienne à la démocratie représentative avait supposé que soit intercalé un échelon de délégation, le passage de la démocratie représentative "classique ", façon $18^{\text {ème }}$ siècle, à la démocratie représentative telle que supposée dans ou par le constitutionnalisme global suppose un nouvel échelon de délégation - non plus cette fois du peuple à ses représentants parlementaires, mais du peuple à ses représentants-juges. Le juge en effet n'est plus ici seulement le " tiers pouvoir » qui tranche des litiges mais bien plus largement, un des acteurs chargé de prendre des décisions dans les matières et dans les termes des politiques publiques ${ }^{35}$. Dans un autre genre (mais pointant dans la même direction), on peut lire les travaux de Pierre Rosanvallon sur la " perte de centralité de l'expression électorale » et la montée en puissance corrélative de nouvelles formes de légitimités qu'il nomme d'impartialité, de réflexivité et de proximité ${ }^{36}$. Ces nouvelles formes de légitimité sont suffisamment importantes pour que Rosanvallon croie pourvoir y voir advenir " un nouveau continent, celui de la démocratie indirecte ", destiné à " compenser et corriger les déficiences de la démocratie électorale représentative ${ }^{37}$.

L'objectif de la présente contribution n'est nullement de discuter ces différentes options théoriques relatives à la fonction des juges ou aux conditions de leur légitimité. II s'agit simplement de suggérer que l'espace théorique existe, dans différents âges et différents courants de la théorie constitutionnelle, tant classique que contemporaine, pour penser les choix politiques effectués par les juges - et donc, pour poser la question de savoir si celles et ceux au nom desquels ils les effectuent devraient pouvoir y prendre part ou être représentés à leur occasion. Car en effet, à mesure que le rôle politique (créateur) du juge est accepté et théorisé par la pensée juridique, l'hypothèse de la responsabilité (accountability) et de la représentativité corrélatives se fraie un chemin. On pourrait en effet considérer que, dans ces conditions de re-théorisation de la justice, les raisons de considérer qu'elle devrait être maintenue à l'écart d'une réflexion globale sur la diversité et la représentativité qui affecte aujourd'hui largement l'ensemble des lieux de pouvoir: si le juge tranche et est, comme d'autres, un acteur politique, au nom de quoi devrait-il demeurer indemne d'interrogations relatives à sa représentativité ?

\footnotetext{
${ }^{34}$ Mathias Kumm, « Institutionalizing Socratic Contestation: the Rationalist Human Rights Paradigm, Legitimate Authority and the Point of Judicial Review », European Journal of Legal Studies, 1 (2), 2007, p. 4 : http://www.ejls.eu/2/26UK.pdf

${ }^{35}$ Mathias Kumm (ibid., p. 14) appelle cela la contestation socratique : "Je soutiens la thèse selon laquelle l'objet du judicial review est d'institutionnaliser la contestation socratique. La contestation socratique désigne l'échange critique avec les autorités, dans le but d'évaluer leurs prétentions et leur fondement sur de bonnes raisons ". Il poursuit en expliquant que d'ailleurs, la capacité au raisonnement juridique n'est pas la qualité la plus importante ou en tous cas la plus éprouvée du juge, puisqu'en toute hypothèse " sous le paradigme des droits de l'Homme, le droit fournit en fait très peu de lignes directrices pour la résolution de cas concrets ". II conclut, dès lors, que " la sagesse spécifique de Socrate et des juges constitutionnels ne tient pas dans ce qu'ils savent au sujet des théories de la justice ou de la politique, mais dans les questions qu'ils savent poser aux autres $"$.

${ }^{36}$ Pierre Rosanvallon, La légitimité démocratique. Impartialité, réflexivité, proximité, Paris, Seuil, 2008, p. 18.

${ }^{37}$ Ibid., p. 24.
} 


\section{B. Qualités du juge dans le paradigme constitutionnaliste : la représentation équilibrée entre les sexes comme garantie des vertus judiciaires?}

Au-delà de la disponibilité d'un cadre théorique permettant de penser une forme de responsabilité (accountability) des juges, on peut en effet observer que les termes concrets dans lesquels s'énonce ou se décline aujourd'hui le standard du "procès équitable " pourraient aisément, eux aussi, permettre de penser comme congruent plutôt qu'antagoniste le rôle des exigences de représentation équilibrée entre les sexes. Deux des standards juridiques aujourd'hui dominants lorsque sont évoquées les vertus d'une bonne justice sont l'impartialité, d'une part, et la théorie des apparences (notamment développée par la Cour européenne des droits de l'Homme) d'autre part. Or ces deux standards semblent pouvoir être aisément interprétés ou réinterprétés comme propices à l'idée d'une représentation équilibrée entre les sexes au sein même de la justice.

\section{Impartialité}

Sally Kenney évoque à ce propos un exemple intéressant : en 2001, un groupe de pression qui milite pour l'égalité entre les sexes soulevait, devant la House of Lords, la question de l'impartialité d'une juridiction devant juger une affaire de viol: "En l'absence de femmes dans la formation de jugement [réunie pour juger une affaire de viol], au moins deux sur cinq, la Cour ne constituerait pas un "tribunal impartial" tel que requis par les traités internationaux relatifs aux droits de l'Homme ", au sens où " un tribunal impartial est une condition nécessaire à la confiance du public en la justice ${ }^{38}$. Certes, cet argument $n^{\prime} a$ pas convaincu les juges suprêmes. II n'en reste pas moins que l'exemple révèle la possibilité de trouver des ressources internes au droit pour fonder l'idée d'une proportion accrue de femmes dans les juridictions. En l'occurrence, il s'agit précisément là d'une perspective qui, par-delà cette affaire R.v.A., semble prise tout à fait au sérieux au Royaume-Uni, à en juger par le rapport de 2012 préconisant une diversité accrue dans la composition de la justice britannique $^{39}$, au motif que : "Les statistiques révèlent qu'en 2011, seuls 5,1\% des juges étaient BAME [Black, Asian and Minority Ethnic] et seulement 22,3\% étaient des femmes. Le comité souligne que la diversité incorpore nombre d'autres éléments, tels que l'aptitude physique, l'orientation sexuelle, la profession juridique et l'origine sociale, et il rejette l'idée que ceux qui proviennent de ces groupes sous-représentés seraient des candidats moins valables ou que l'objectif d'une justice plus diverse serait de nature à fragiliser la qualité de nos juges ". Cette perspective liant exigences d'impartialité de la justice et de diversité dans la composition des juridictions a trouvé un nouvel écho dans un autre rapport relatif à la

\footnotetext{
${ }^{38}$ Sally Kenney, "Equal Employment Opportunity and Representation : Extending the Frame to Courts », Social Politics, 11 (1), 2004, p. 102. Elle explique plus en plus loin : "La Fawcett Society, issue du mouvement pour le suffrage féminin, a fait le lien entre ses campagnes pour élire plus de représentantes au Parlement et la représentation des femmes au sein de la magistrature. Je crois que c'est le premier effort d'une organisation de femmes qui ne fait pas partie de la profession juridique pour faire une telle demande. Un pas de géant a été franchi pour qu'un groupe de femmes en dehors de la profession juridique passe de la critique des juges sexistes à une demande de justice représentative, comme le montre l'importance croissante que l'Alliance nationale accorde à la CPI en Europe et à l'international, ainsi que la nouvelle attention portée à la composition des tribunaux".

${ }^{39}$ http://www.publications.parliament.uk/pa/ld201012/Idselect/ldconst/272/272.pdf
} 
justice britannique : le Lammy Review ${ }^{40}$ affirme ainsi dès l'introduction : " La confiance dans les décisions impartiales repose non seulement sur l'indépendance constitutionnelle de la magistrature, mais aussi sur le lien entre les tribunaux et les communautés qu'ils servent ${ }^{41}$. Au-delà du Royaume-Uni, on voit cette question de la représentativité liée aux vertus contemporaines d'une bonne justice s'exprimer dans différents rapports, à l'instar de la récente étude menée conjointement par Open Society Justice Initiative et la Commission internationale des juristes sur les juges et experts en matière de droits humains ${ }^{42}$. Où l'on voit que l'impartialité peut bien être comprise comme requérant une forme de diversité ${ }^{43}$.

\section{La théorie des apparences}

La théorie des apparences a été développée par la Cour européenne des droits de l'Homme précisément en matière de justice; selon cette théorie, la justice ne doit pas seulement être rendue de manière impartiale, elle doit aussi être rendue d'une manière apparemment impartiale (dans sa formulation anglaise : not only must Justice be done; it must also be seen to be done $e^{44}$ ).

Il est intéressant de se pencher sur les fondements et les justifications de cette théorie des apparences. Pourquoi la Cour européenne des droits de l'Homme considère-telle que l'impartialité seule ne suffit pas; et qu'au-delà, il importe encore que les apparences sous lesquelles la justice est rendue véhiculent la notion d'impartialité ? II y a fort à parier que des notions comme celle de l'acceptabilité sociale de la justice, clé nécessaire sinon suffisante de son autorité, joue un rôle déterminant dans cette théorie. Or on peut noter à ce sujet que, au moins pour ce qui est des juridictions autres que les juridictions ordinaires (c'est-à-dire, notamment, pour les juridictions constitutionnelles et internationales), il est très généralement admis que des facteurs tels que la nationalité ou l'expérience politique passée sont des facteurs importants : ils jouent précisément un rôle crucial du point de vue de l'acceptabilité de ces formes de justice.

Le critère de la nationalité irrigue, c'est certain, les règles de compositions comme l'idée de légitimité de la justice internationale. Dans son étude consacrée à la question du genre à la Cour de Justice de l'Union européenne, Sally Kenney s'est notamment penchée sur les débats qui y ont eu lieu après que la Cour ait commencé à siéger dans des formations réduites à trois ou cinq juges. Elle cite les propos tenus alors par le président de la Cour, Mackenzie Stuart : « Afin d'emporter la conviction, tant vis-à-vis des requérants que vis-à-vis des Etats membres, il importe que toute décision de cette Cour émane d'un corps qui représente un spectre suffisamment large de pensée juridique. Or une Cour de trois membres est, aux yeux de nombreuses personnes, trop restreinte ${ }^{45}$. Mais il est bien d'autres critères qui se voient semblablement assigner un rôle important dans la fabrication

\footnotetext{
40 https://www.gov.uk/government/uploads/system/uploads/attachment data/file/643001/lammy-reviewfinal-report.pdf

${ }^{41}$ Ibid., p. 31.

${ }^{42}$ Open Society Justice Initiative et de la Commission Internationale des juristes : Strengthening from within : Law and Practice in the Selection of human rights judges and commissioners, 2017 : https://www.icj.org/wpcontent/uploads/2017/11/Universal-Strengthening-from-Within-Publications-Reports-2017-ENG.pdf

${ }^{43}$ V. aussi lyiola Solanke, "Diversity and Independence in the European Court of Justice ", Columbia Journal of European Law, 15 (1) 2009, vol. 15, n¹, p. 89-121.

${ }^{44}$ Il s'agit d'un extrait d'un arrêt britannique très célèbre relatif, précisément, à l'impartialité du juge : $R v$ Sussex Justices, Ex parte McCarthy ([1924] 1 KB 256, [1923] All ER 233.

${ }^{45}$ Sally Kenney, Gender and Justice, op. cit., souligné par moi.
} 
ou la garantie de la légitimité juridictionnelle. Diverses cours connaissent ainsi des règles de quotas. Ce sont parfois des quotas par sexe (il en existe en Autriche ou en Belgique pour la composition de la cour constitutionnelle), et parfois des quotas linguistiques (la Cour Suprême du Canada doit comprendre au moins trois juges ou avocats québecois afin de représenter le Canada francophone). Aux Etats-Unis, une attention particulière est parfois portée à la diversité ethnique des juges. II en va ainsi en Floride, où il est prévu que " lorsqu'il procède à une nomination, le gouverneur doit prêter attention, dans la mesure du possible, à ce que la commission [qui propose les candidats] reflète la diversité raciale, ethnique et de genre, ainsi que la distribution géographique de la population au regard de la juridiction territoriale de la cour dont il s'agit ${ }^{46}$; mais aussi dans le système judiciaire fédéral : "Les commissions de sélection au mérite établies par la section 631 b5 du titre 28 du US Code doivent prendre en considération l'ensemble des personnes qualifiées dans les recommandations qu'elles font pour les nominations à la District Court, et en particulier les groupes tels que celui des femmes, des Noirs, des Hispaniques et autres minorités... ". Plus classiquement encore - même si ce critère-là demeure généralement implicite -, c'est souvent le capital politique qui joue un rôle déterminant pour la nomination dans une juridiction international ou suprême.

On comprend dès lors la logique ici suggérée : si la théorie des apparences vise en effet à entretenir la légitimité sociologique des cours et juridictions, elle peut bien alors constituer, elle aussi, un cadre argumentatif favorable aux revendications visant une représentation équilibrée des hommes et des femmes au sein du pouvoir judiciaire.

Est-ce que le genre de la justice (son identité de genre, mais aussi au-delà, de race, classe, religion, langue, etc.) est une question qui mérite d'être examinée dans une réflexion sur l'indépendance de la justice? II me semble que oui; voilà la seule affirmation de la présente contribution. Et il importe de ne pas se méprendre sur ses contours. C'est bien parce que "le Droit" n'est pas cette instance normative neutre et objective (mais bien plutôt le résultat contingent et toujours renégocié de divers rapports de force sociaux), et parce que "les cours" ne sont pas des entités abstraites auxquelles n'accèdent que les meilleurs d'entre nous sur la seule considération du mérite et de la compétence, qu'il vaut la peine de s'interroger sur la place des femmes (et d'autres) dans la justice. Car en effet, ce qui compte comme " objectif ", " impartial » ou " neutre " dépend très largement du point de perspective que l'on adopte. En ce sens, l'interrogation n'est pas l'habillage malhabile d'une pensée essentialiste qui voudrait qu'il importe que des femmes siègent dans les juridictions pour que leurs intérêts ou leur(s) cause(s) soient mieux entendus. Bien différemment, elle entend seulement porter sur le sens que nos ordres juridiques confèrent au principe d'égalité. Acceptant pleinement qu'« il n'y a pas de jugement "pur", libéré des conditions physiques de sa réalisation, pas plus qu'il n'existe de droit désincarné, en apesanteur sociale", on entend suggérer que "la vertu ne consiste pas à refouler ces déterminismes, mais au contraire à les assumer en acceptant la finitude du jugement judiciaire. Elle n'est pas la négation vertueuse de ces influences inévitables sur la décision du juge mais leur reconnaissance et leur mise à distance ${ }^{47}$.

\footnotetext{
${ }^{46}$ Fla Stat. Ann $§ 43.291$ (2005) : section 43.291.

${ }^{47}$ Anatoine Garapon, Julie Allard, Frédéric Gros, Les vertus du juge, Paris, Dalloz, 2008, p. 6-8.
} 